\title{
A UTILIZAÇÃO DE MEDICAMENTOS HOMEOPÁTICOS NA REGIÃO METROPOLITANA DE CURITIBA
}

\section{HOMEOPATHIC MEDICINES UTILIZATION AT CURITIBA METROPOLITAN REGION}

\author{
OLIVEIRA, A. B. *1,4; ZANIN, S. M. W. ${ }^{2,4}$; MIGUEL, M. D. ${ }^{3,4}$
}

\footnotetext{
1. Acadêmica do curso de Farmácia Bioquímica e Industrial - UFPR

2. Mestre em Bioquímica - UFPR, Disciplina de Farmacotécnica Homeopática *Email: sandraz@ufpr.br>

3. Mestre em Educação PUC-PR, Doutora em Prod. Vegetal - UFPR, Disciplina de Farmacotécnica

4. Projeto de Pesquisa: Atenção Farmacêutica na Formação do Acadêmico de Farmácia
}

Recebido em: 09/2004 Aprovado em: 10/2004

\begin{abstract}
RESUMO
A Homeopatia é uma opção terapêutica que inclui o tratamento medicamentoso individualizado e unicista, com apresentação de efeitos indesejáveis nula devido ao grau de diluição infinitesimal. Estas características, junto à verificação de melhora e cura do estado patológico do paciente, têm promovido o aumento da utilização da Homeopatia. Com o objetivo de verificar a amplitude do uso desta terapêutica, realizou-se pesquisa em farmácias de manipulação homeopática de Curitiba e Região Metropolitana, buscando detectar quais os medicamentos mais solicitados pelos médicos, assim como formas farmacêuticas e escalas de diluição infinitesimal. A percepção da predominância do uso de policrestos remete à importância da presença do farmacêutico na farmácia homeopática. Este com função de esclarecimento e orientação ao paciente no que diz respeito ao tratamento e cuidados com os medicamentos, os promotores da cura. Além do acompanhamento do tratamento ao lado do médico, mantendo troca de informações com o mesmo, o que permite a otimização do atendimento farmacêutico dentro do conceito de promotor de saúde.

Palavras-chave: Homeopatia; Amplitude de tratamento; Medicamentos homeopáticos; Farmacêutico na promoção da saúde.
\end{abstract}

\begin{abstract}
Homeopathy is a therapeutic option that includes individual and unicist medicinal treatment. Since it can cure or improve patient health, its use has increased over the years. A research was realized in Homeopathic Manufacturing Pharmacies in the greater Curitiba area with the objective of verifying its utilization extent. In addition, the goal was to detect what homeopathic medicines are the most used by doctors, with its pharmaceutical presentations and dilution scale. Policrest was the most predominantly used treatment prescribed by homeopathic pharmacies. Treatment was accompanied by doctors, and the patient-doctor exchange information was crucial for treatment. That allowed a pharmaceutical service optimization under the health promoter concept.

key words: Homeopathy, Treatment extent, Homeopathic medicines, Pharmacist on health promotion.
\end{abstract}

\section{INTRODUÇÃO}

A Homeopatia é uma terapia fundamentada no princípio da similitude, explicado pela teoria que a cura decorre da reação secundária do organismo à substância farmacológica, o chamado efeito rebote, num esforço para manter a homeostase (MORAIS, 2002). O mecanismo de defesa age de modo sistemático e integrado, nos níveis físico, emocional e mental, mantendo o equilíbrio do organismo (CAIRO, 1979). Tal terapia apóia-se na observação experimental de que toda substância capaz de provocar determinados sintomas em uma pessoa sadia, é capaz de curar estes mesmos sintomas em uma pessoa enferma. Nesse contexto, a Homeopatia é um sistema científico bem definido, com metodologia de pesquisa própria, reconhecida como tal pelos Conselhos Federais de Medicina e Farmácia desde a década de 1980.

Trata-se de uma terapêutica relativamente atóxica, que tem se mostrado eficiente; de baixo custo, o que idealiza a utilização por grande parte da população de baixo nível sócio-econômico e escolar, possibilitando o atendimento homeopático pelo SUS (BRANDAO, 
2001); e de fácil administração por crianças, idosos e adultos de ambos os sexos, além de animais. Atualmente existem veterinários e cirurgiões-dentistas utilizando homeopatia em suas clínicas, assim sendo uma terapêutica de interação com todas as especialidades médicas. A lei da similitude é o motivo pelo qual a Homeopatia leva em consideração a individualidade dos pacientes para tratá-los, conduzindo ao tratamento unicista (AFHERJ), evitando o uso exagerado e muitas vezes inadequado de diferentes medicamentos para o mesmo diagnóstico. Entretanto, conforme o caso, os tratamentos alopáticos e homeopáticos, além de outros denominados "alternativos", não são incompatíveis, o seu uso depende das necessidades do paciente (MORAIS, 2002); qual fato sugere que os profissionais da área da saúde devem possuir formação que domine todas as correntes médicas.

O medicamento administrado é preparado de modo diluído (doses infinitesimais) (AFHERJ) que impede efeitos adversos indesejáveis ou intoxicações a quem se expuser a overdose ou a troca de medicação (LOCKIE, 2000), porém possui capacidade terapêutica devido à técnica de dinamização, por meio da qual a energia curativa latente na substância bruta é liberada, passando a agir no paciente (AFHERJ). A eficiência do medicamento homeopático é verificada experimentalmente, conforme seu uso, levando à cura. A constatação científica do seu mecanismo de ação inicia-se atualmente, com base em postulados que indicam a formação de grupamentos moleculares da água em soluções cujas diluições dinamizadas de substâncias orgânicas e inorgânicas ultrapassem o número de Avogadro. Tais grupamentos são visualizados por meio de microscopia eletrônica, possuem número variável de unidades moleculares constituintes e replicam-se a cada diluição; apresentam características específicas que dizem respeito a propriedades elétricas, adesivas e estímulo de células do sistema imunológico in vitro (MORAIS, 2002).

As pesquisas demonstram que as soluções homeopáticas não são solvente comum, mas líquidos alterados em suas estruturas que realmente podem modificar tecidos, órgãos e todo o corpo, revelando assim uma atividade biológica que ainda está para ser farmacologicamente definida. Tais descobertas sugerem a efetividade já detectada há muitos anos dos medicamentos homeopáticos, cujo uso tem sido mais aceito e divulgado atualmente.

\section{METODOLOGIA}

Com o objetivo de verificar a amplitude do tratamento por Homeopatia na região metropolitana de Curitiba, realizou-se um levantamento dos medicamentos homeopáticos mais indicados sob receita médica, por meio de pesquisa promovida com base no Livro de Registro dos medicamentos manipulados de quinze farmácias homeopáticas da localidade. Os dados obtidos foram submetidos a análise percentual.

\section{RESULTADOS E DISCUSSÃO}

A partir dos resultados relacionados na tabela 1 e gráfico 1, verifica-se que os medicamentos mais solicitados pelos médicos homeopatas (Pulsatilla, Sulphur, Nux vomica, Natrum muriaticum, Lycopodium, Calcarea carbonica, Lachesis mutta, Sepia, Arsenicum album, Silicea, Arnica montana) pertencem às classes policrestos e semipolicrestos, medicamentos homeopáticos que atingem o maior número de sintomas nos experimentos clínicos em homens sãos, que apresentam reprodutibilidade. Quais são utilizados em um 
contexto geral, de acordo com os tipos constitucionais e personalizados dos pacientes, para tratamento de doenças crônicas ou em caráter preventivo (LOCKIE, 2000; LOCKIE, GEDDES, 2001).

TABELA 1 - OS MEDICAMENTOS HOMEOPÁtICOS MAIS UtILIZADOS NA REGIÃO MEtrOPOLITANA DE CURITIBA

\begin{tabular}{cc}
\hline MEDICAMENTOS & $\%$ DE USO \\
\hline Belladona & $8,14 \%$ \\
Sulphur & $7,38 \%$ \\
Nux vomica & $7,12 \%$ \\
Natrum muriaticum & $6,11 \%$ \\
Lycopodium & $5,60 \%$ \\
Phosphorus & $5,60 \%$ \\
Calcarea carbonica & $5,34 \%$ \\
Lachesis & $4,83 \%$ \\
Sepia & $3,82 \%$ \\
Arsenicum album & $3,05 \%$ \\
Silicea & $2,80 \%$ \\
Arnica montana & $2,80 \%$ \\
Outros & $29,27 \%$ \\
\hline
\end{tabular}

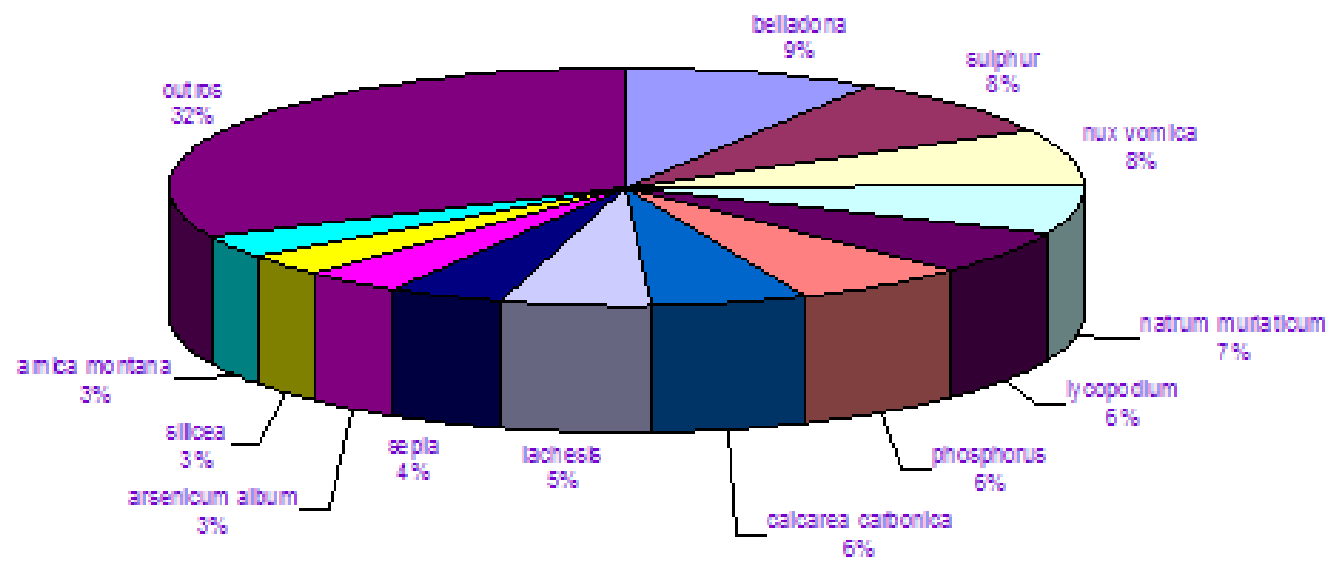

GRÁFICO 1 - Os Medicamentos Homeopáticos mais Utilizados na Região Metropolitana de Curitiba

Tais resultados sugerem que o tratamento homeopático ocorre em sua grande maioria para doenças de tratamento dificultado, como as degenerativas e de longo prazo; possivelmente em conseqüência do tratamento alopático inefetivo ou com efeitos adversos indesejáveis pelo paciente, conforme já perceptível na prática, de maneira não formalizada. Presume-se que o tratamento predominante com policrestos seja motivado pela própria avaliação unicista dos clínicos homeopatas para levar à cura um alto índice de perturbações do paciente, o que também possibilita a administração de tais medicamentos com finalidade preventiva.

A homeopatia ainda possui a vantagem de apresentar diversas formas farmacêuticas para o mesmo medicamento, o que facilita a administração por diversas classes de indivíduos, como por exemplo para uso pediátrico, adulto e veterinário. Na tabela 2 e no gráfico 2, está representada a relação de formas farmacêuticas mais prescritas aos usuários das farmácias onde foi realizada a pesquisa. A forma líquida em gotas é a mais solicitada, em $34,8 \%$ das formulações; seguida pela dose única, em $28,5 \%$, sugerindo o uso de 
medicamento homeopático pelas diversas faixas etárias e animais, visto que a prescrição líquida pode ser administrada por qualquer destes indivíduos. A baixa utilização de formas farmacêuticas de uso tópico (cremes, loções, gel, soluções nasais / otológicas, óvulos vaginais, entre outras), remete à característica homeopática do tratamento do ser humano como totalidade, em lugar da demonstração sintomática, crônica ou aguda, em si. O uso das formas tópicas ocorre em concomitância com as formas sistemáticas.

TABELA 2 - As formas farmacêuticas mais prescritas para medicamentos homeopáticos na região metropolitana de Curitiba

\begin{tabular}{cc}
\hline MEDICAMENTOS & $\%$ DE USO \\
\hline Líquido em gotas & $34,80 \%$ \\
Líquido em dose única & $28,54 \%$ \\
Glóbulos & $17,76 \%$ \\
Cremes / Loções & $8,30 \%$ \\
Tabletes & $6,08 \%$ \\
Pomadas & $2,62 \%$ \\
Pós (papelotes) & $0,50 \%$ \\
Comprimidos & $0,27 \%$ \\
Outros & $1,23 \%$ \\
\hline
\end{tabular}

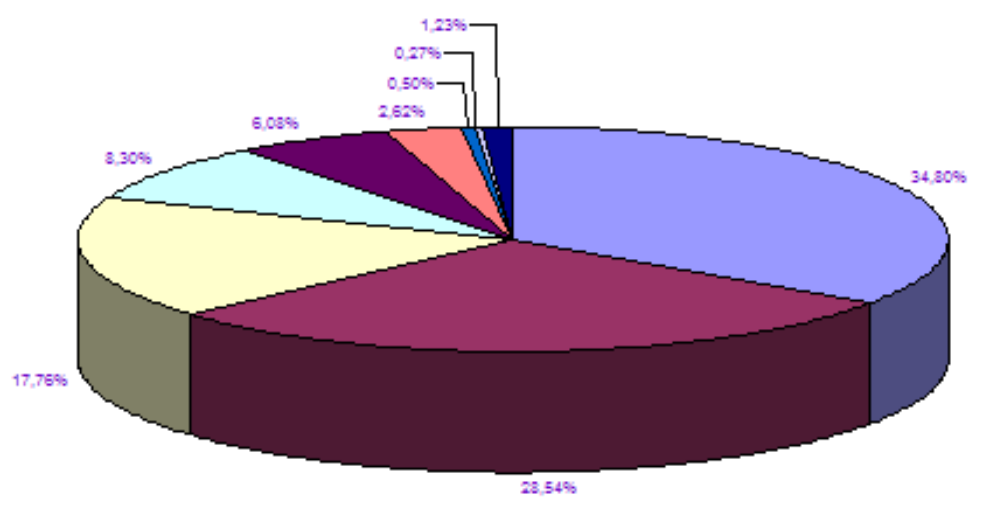

\begin{tabular}{|lll|}
\hline$\square$ liquido em gotas & Qliquido em dose única & $\square$ glóbulos \\
$\square_{\text {cremes / loçốes }}$ & $\square_{\text {tabletes }}$ & $\square_{\text {pomadas }}$ \\
$\square_{\text {pós (papelotes) }}$ & $\square_{\text {comprimidos }}$ & $\square_{\text {outros }}$ \\
\hline
\end{tabular}

GRÁFICO 2 - As Formas Farmacêuticas mais Prescritas para Medicamentos Homeopáticos na Região Metropolitana de Curitiba

Considerando-se a individualidade dos medicamentos, a definição da potência mais utilizada é dificultada, porém verificou-se a frequência da solicitação das diversas escalas de ultradiluição. Como apresentado na tabela 3, gráfico 3, a escala de ultradiluição mais solicitada é a centesimal hahnemanniana $(\mathrm{CH})$, em 36,3\% das prescrições, sendo que a escala fluxo contínuo (FC) tem utilidade para potências centesimais mais altas e foi verificada em $26,4 \%$ das formulações; a escala "special dinamization" (SD) foi solicitada em 32,8\% das receitas; a escala cinqüenta-milesimal (LM) foi notada em 3,6\% das formulações, a escala decimal (D) é verificada em 0,75\% das solicitações; e a korsakoviana (K) é a menos utilizada, em 0,23\% das formulações. Tais resultados refletem a escolha da escala pelo clínico considerando a maior potência que o medicamento terá ao fim das diluições e 
dinamizações: a escala CH permite uma diluição de 1:100 e cem sucussões a cada potencialização; a FC geralmente permite uma diluição de 1:100 e vinte sucussões a cada potencialização; a SD encerra uma diluição de 1:100000 e 200 sucussões a cada potencialização (apresenta maior potência mas não é uma metodologia clássica, é uma escala recentemente postulada); a LM segue técnica especial de preparo e 100 sucussões a cada potencialização; a D apresenta diluição 1:10 e 100 sucussões a cada potencialização; enquanto a K apresenta diluição de aproximadamente 1:100 e 100 sucussões. Considera-se que a potência medicamentosa aumenta tanto maior é a diluição e o número de sucussões durante a dinamização.

TABELA 3 - As Escalas de Ultradiluição Homeopática mais Solicitadas na Região Metropolitana de Curitiba

\begin{tabular}{cc}
\hline ESCALAS & $\%$ \\
\hline CH & $36,30 \%$ \\
FC & $26,45 \%$ \\
SD & $32,80 \%$ \\
LM & $3,60 \%$ \\
D & $0,75 \%$ \\
K & $0,23 \%$ \\
\hline
\end{tabular}

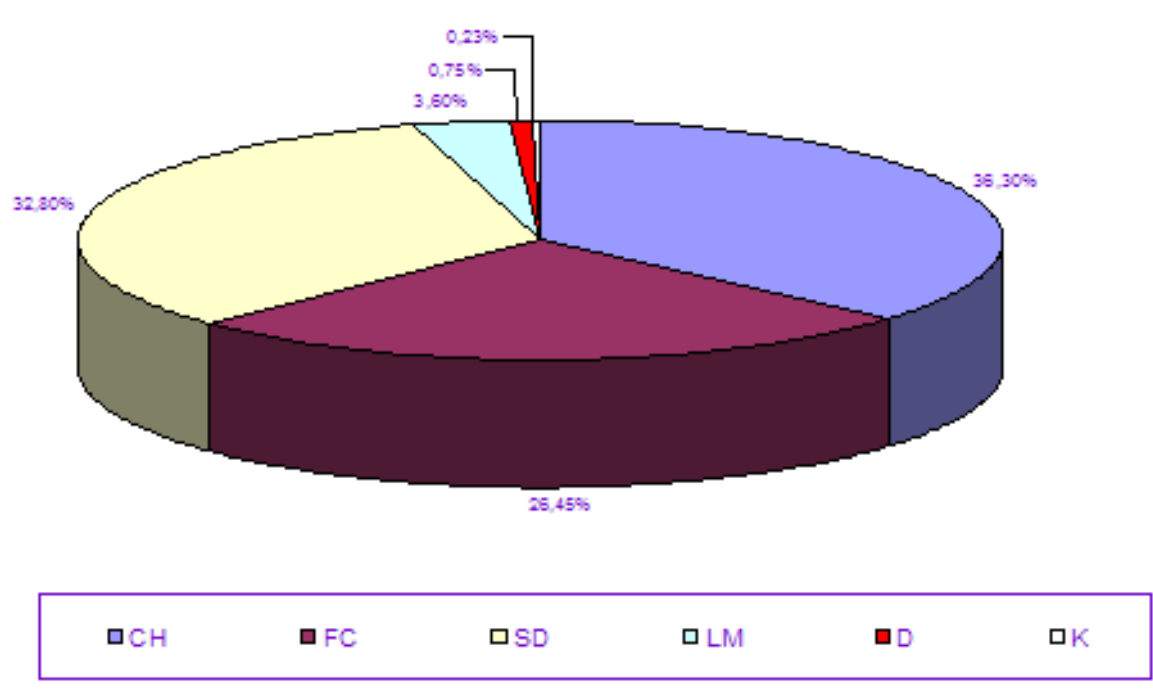

GRÁFICO 3 - As Escalas de Ultradiluição Homeopática mais Solicitadas na Região Metropolitana de Curitiba

\section{CONSIDERAÇÕES}

Atualmente, o tratamento homeopático é motivado sobretudo por atrativos que levam à melhoria da qualidade de vida. Ao contrário da medicina convencional, a homeopatia não é uma terapia invasiva, dispensando cirurgias, exames incômodos e drogas que provocam efeitos colaterais quase sempre perigosos. Sua abordagem é sistêmica e tem como foco o paciente à doença em si, muito mais humanitária e personalizada que na medicina alopática, cujos diagnósticos dependem hoje da tecnologia que da acuidade do médico (MORAIS, 2002). Porém, há que se tomar cuidados com o promotor da cura, o medicamento, para garantir a efetividade ao tratamento; remete então à importância da influência farmacêutica inserida no contexto homeopático. 
Durante a dispensação, o farmacêutico homeopata deve informar o paciente quanto aos cuidados que deverão ser tomados para a utilização e conservação adequados do medicamento homeopático e quanto às demais situações que possam contribuir para a melhoria e manutenção de sua saúde (ABFHRJ; OLIVEIRA; 2001; OMS, 1993). Dentre as orientações, devem incluir-se características do local de armazenamento do medicamento (evitar exposição a umidade, luz, calor e aparelhos que emitem radiações, entre outros), do cumprimento das recomendações do clínico quanto ao horário e concomitância da administração, e a consciência da procura de orientação profissional em caso de mudança de estado clínico ou dúvidas do paciente (OLIVEIRA, 2001). O esclarecimento da farmácia em relação a homeopatia deve ser procedido por meio de explanações do profissional farmacêutico (PERETTA, 2000) ou pela distribuição gratuita do Manual do Consumidor de Homeopatia concedido pela Associação Brasileira de Farmacêuticos Homeopatas (ABFH) (ABFHERJ). A automedicação em homeopatia também é de extrema importância dentro do tratamento pois o medicamento homeopático, além de ser específico, apresenta ação farmacológica ao estimular os mecanismos de defesa do organismo (MORAIS, 2002). Cabe ao farmacêutico homeopata evitá-la, expondo ao paciente a periculosidade de recomendar medicamentos a outros indivíduos e de administrá-los sem orientação do clínico.

Antes de proceder a orientação, porém, o farmacêutico deverá obter informações importantes sobre o paciente, tais como idade, sexo, seguimento de outras condutas terapêuticas, apresentação de doença crônica ou vícios, compreensão sobre medicamento homeopático, situação da alimentação e qualidade de vida (FURTADO, 2000). Estas e outras condições do usuário são muito importantes para a função do farmacêutico como promotor de saúde integral, de modo que os componentes técnicos e humanísticos se harmonizam em torno do paciente (OMS, 1993). Além disso, de posse de tais dados, o farmacêutico homeopata poderá otimizar o tratamento entrando em contato com os clínicos para esclarecer dúvidas, propor soluções técncas e fornecer informações sobre o medicamento homeopático, realizando a denominada entrevista médica, na qual a relação médicofarmacêutico-paciente se completa (BRANDÃO, 2001). A intervenção farmacêutica ganha importância na dificuldade médica de prescrever o medicamento correto, visto que levam em consideração a totalidade dos sintomas, incluindo que muitos pacientes escondem alguns sintomas.

Tendo em vista as exposições, nota-se que o trabalho efetivo do farmacêutico homeopata traz garantia de tratamento bem direcionado, de acordo com o previsto pelo clínico homeopata.

\section{CONCLUSÃO}

A utilização ampliada da Homeopatia, verificada pela pesquisa realizada, remete à eficiência do âmbito profissional como promotor de saúde, baseando-se na orientação e interação entre clínico, farmacêutico e pacientes; para evitar que haja com os medicamentos homeopáticos as falhas graves atualmente existentes no manuseio dos medicamentos alopáticos. Para tanto, faz-se necessária uma maior concentração sobre os usuários por parte dos profissionais de saúde especializados em Homeopatia, em atitudes e esclarecimentos, além da percepção de mudanças do estado geral do paciente. Essas propriedades devem fixar-se na atuação profissional, desde a sua formação (MIGUEL, 2000). 
O farmacêutico homeopata está diretamente inserido nesse contexto, não somente no que diz respeito à efetuação correta das atividades da farmácia homeopática, garantindo a qualidade dos medicamentos usados e conforme regulamentação vigente: a Lei 5991/73 (MINISTÉRIO DA SAUDE DO BRASIL, 1973) afirma que " a farmácia homeopática só poderá manipular fórmulas oficinais e magistrais, obedecida a farmacotécnica homeopática"; a RDC 33/01 (MINISTÉRIO DA SAÚDE DO BRASIL, 2001) fixa os requisitos mínimos exigidos para atividades que envolvam a produção, manutenção, transporte e dispensação de fórmulas magistrais e oficinais aliado outros produtos de interesse para a saúde, conforme as Boas Práticas de Manipulação; cujas infrações são sujeitas às penalidades previstas na Lei 6437/ 77 (MINISTÉRIO DA SAÚDE DO BRASIL, 1977). O profissional também deve orientar o usuário na perspectiva da promoção de saúde, quanto ao manuseio dos medicamentos e ao esclarecimento de dúvidas. Desse modo, evitando sobremaneira erros terapêuticos devido à negligência profissional e à desinformação por parte do paciente.

\section{REFERÊNCIAS}

AFHERJ. Manual do Consumidor de Homeopatia. Rio de Janeiro : Informativos da ABFH - Associação Brasileira de Farmacêuticos Homeopatas; 2003.

BRANDÃO, A. Homeopatizando. Pharmacia Brasileira n. 28, set/out 2001. Brasília : Conselho Federal de Farmácia; 2001.

CAIRO, N. Guia de Medicina Homeopática. 21 ed. São Paulo : Editora Teixeira; 1979.

FURTADO, G. R. A Energia da Atenção Farmacêutica. Pharmacia Brasileira n. 23, nov/dez 2000. Brasilia : Conselho Federal de Farmácia; 2000.

LOCKIE, A. Enciclopédia de Homeopatia. Lisboa : Livraria Civilização Editora; 2000.

LOCKIE, A; GEDDES, N. Guia Completo de Homeopatia. São Paulo : Editora Ática; 2001.

MINISTÉRIO DA SAÚDE DO BRASIL. Diário Oficial [da] República Federativa do Brasil. Brasília : Lei 5991 de 17 de dezembro de 1973.

MINISTÉRIO DA SAÚDE DO BRASIL. Diário Oficial [da] República Federativa do Brasil. Brasilia : Lei 6437 de 20 de agosto de 1977.

MINISTÉRIO DA SAÚDE DO BRASIL. Diário Oficial [da] República Federativa do Brasil. Brasília : Resolução da Diretoria Colegiada 33 de 19 de abril de 2000.

MIGUEL, M. D. Atenção Farmacêutica na Formação do Acadêmico de Farmácia. Curitiba : Núcleo Interdisciplinar de Pesquisa em Ciências Farmacêuticas; set/2000. 46p.

MORAIS, JOMAR. O Poder das Bolinhas. Revista Superinteressante n. 172, janeiro de 2002. São Paulo : Editora Abril; 2002.

OLIVEIRA, A. B.; MIGUEL, M. D.; ZANIN, S. M. Uma Análise da Atenção Farmacêutica. Infarma v. 13, n.9/10. São Paulo; 2001.

OMS-OPS. El Papel del Farmacéutico en el Sistema de Atención de Salud. Informe de la reunión de la OMS (OPS/HSS/ HSE/95,01). Tokio; 1993.

PERETTA, M; CICCIA, G. Reengenharia Farmacêutica - Guia para Implantar Atenção Farmacêutica. Brasília : Ethosfarma; 2000. 